\title{
Hongos detectados en la entomofauna asociada a Espeletia pycnophylla
}

\section{Fungi detected in insects associated to Espeletia pycnophylla}

\author{
Eliana Galíndez-Chicaíza'; Luz Estela Lagos-Mora²; Guillermo Castillo-Belalcázar; \\ Claudia Salazar-González ${ }^{4}$; Carlos Betancourth-García ${ }^{5}$
}

Bióloga, Universidad de Nariño. Pasto - Nariño, Colombia; e-mail: elianamg3@gmail.com; Dhttps://orcid.org/0000-0001-8209-8133

²Bióloga, M.Sc., Universidad de Nariño. Pasto - Nariño, Colombia; e-mail: luzestela@udenar.edu.co; Dhttps://orcid.org/0000-0002-5152-3413

${ }^{3}$ Lic. Biología y Química, Esp. Universidad de Nariño. Pasto - Nariño, Colombia; e-mail: gacastillob@gmail.com; Dhttps://orcid.org/0000-0002-5954-1632

${ }^{4}$ Ing. Agrónomo, M.Sc., Ph.D. Universidad de Nariño, Pasto - Nariño, Colombia; e-mail: claudiasalazarg@yahoo.com; (Dhttps://orcid.org/0000-0002-54612761

${ }^{5}$ Ing. Agrónomo, M.Sc., Ph.D. Universidad de Nariño. Pasto - Nariño, Colombia; e-mail: cbet70@yahoo.com; Dhttps://orcid.org/0000-0001-6573-4230

Cómo citar: Galíndez-Chicaíza, E.; Lagos-Mora, L.E.; Castillo-Belalcázar, G.; Salazar-González, C.; Betancourth-García, C. 2020. Hongos detectados en la entomofauna asociada a Espeletia pycnophylla. Rev. U.D.C.A Act. \& Div. Cient. 23(2):e1497. http://doi. org/10.31910/rudca.v23.n2.2020.1497

Artículo de acceso abierto publicado por Revista U.D.C.A Actualidad \& Divulgación Científica, bajo una licencia Creative Commons CC BY-NC 4.0

Publicación oficial de la Universidad de Ciencias Aplicadas y Ambientales U.D.C.A, Institución de Educación Superior Acreditada de Alta Calidad por el Ministerio de Educación Nacional.

Recibido: Marzo 10 de 2020 Aceptado: Agosto 28 de 2020 Editado por: Helber Adrián Arévalo Maldonado

\section{RESUMEN}

Algunas especies del género Espeletia sp. (Asteraceae) son afectados por hongos fitopatógenos e insectos en páramos de los Andes del Norte, amenazando su permanencia y la prestación de servicios que se les atribuye, como la regulación del ciclo hídrico. La escaza información sobre la afectación en el páramo de Paja Blanca (Nariño, Colombia), dificulta la comprensión de la dinámica del fenómeno y la formulación de estrategias de manejo. El objetivo de esta investigación fue identificar cambios en la composición de las comunidades de insectos asociados a E. pycnophylla durante el declive de las poblaciones de frailejones y evaluar si reflejan modificaciones en los hongos, asociados a dichos insectos. Para ello, se colectaron insectos en plantas de E. pycnophylla sanas y afectadas, a los que se aplicó índices ecológicos, para identificar posibles modificaciones en la diversidad y la composición; además, se aislaron e identificaron morfológica y molecularmente hongos, a partir de estructuras corporales de los insectos. Como resultado, se identificó una diversidad de insectos considerable y que no hay modificación en su composición de insectos entre los dos estados de frailejón analizados. Se logró aislar hongos, como Fusarium oxysporum, Botrytis sp., Epicoccum nigrum, Cladosporium sp., a partir de estructuras de los géneros de insectos Neomyopites sp., Diabrotica sp., Bradysia sp. y Dyscolus sp. Este estudio aporta información sobre la entomofauna presente en E. pycnopylla del páramo de Paja Blanca y hongos asociados a estructuras corporales, como el canal alimentario y aparato bucal de los insectos.

Palabras clave: Canal alimentario; Aparato bucal; Fitosanidad; Hongos fitopatógenos; Asteraceae; Frailejón. 


\section{ABSTRACT}

Some species of the genus Espeletia sp. (Asteraceae) are affected by phytopathogenic fungi and insects in the paramos of the Northern Andes, threatening their permanence and the provision of services attributed to them such as the regulation of the water cycle. The scarce information about the impact on the Paja Blanca páramo (Nariño, Colombia) makes it difficult to understand the dynamics of the phenomenon and the formulation of management strategies. The goal of this research was to identify changes in the composition of the insect communities associated to E. pycnophylla during the decline of the frailejon populations and to evaluate if these reflect modifications in the fungi associated with those insects. For this, insects were collected in healthy and affected E. pycnophylla plants to which ecological indices were applied to identify possible changes in diversity and composition, Also fungi were isolated and identified morphologically and molecularly from body structures of insects. As result, a considerable diversity of insects was identified and no modification in their composition of insects between the two states of frailejón were found. It was possible to isolate fungi such as Fusarium oxysporum, Botrytis sp., Epicoccum nigrum, Cladosporium sp. from structures of the insect genera Neomyopites sp., Diabrotica sp., Bradysia sp. and Dyscolus sp. This study provides information about the entomofauna present in E. pycnopylla from the Paja Blanca páramo and fungi associated with body structures such as the alimentary canal and the oral system of insects.

Keywords: Alimentary canal; Oral apparatus; Phytosanitary; Phytopathogenic fungi; Asteraceae; Big monk.

\section{INTRODUCCIÓN}

Los brotes de enfermedades fúngicas y el ataque de insectos conducen al detrimento en la calidad de los bienes y servicios que las plantas ofrecen, bajo condiciones estables (Nárdiz \& De Cal, 2006). Es por ello, que desde el 2010, se presta especial atención al estado fitosanitario de las poblaciones de frailejón Espeletia sp. (Asteraceae), presentes en los ecosistemas paramunos andinos, debido a que reportan síntomas, como deformación de lámina foliar, clorosis, pudrición radicular y necrosis que, posiblemente, estén asociados con la presencia de hongos fitopatógenos y a la herbivoría por parte de insectos de los órdenes Lepidoptera y Coleoptera (Salinas et al. 2013; Varela, 2014) y que pueden poner en riesgo la prestación de servicios ecosistémicos, como la regulación hídrica.

En 2017 surge el primer reporte de síntomas en frailejones del sur del país, en el páramo de Paja Blanca (Nariño), en la especie Espeletia pycnophylla; sin embargo, no se cuenta con información sobre la entomofauna asociada a esta especie de frailejón, previo a la aparición de los síntomas, hecho que dificulta determinar si ha ocurrido o no una modificación en la composición de insectos, que pueda estar relacionada con la actual condición de E. pycnophylla; además, se desconoce si las especies de hongos presentes en el cuerpo de los insectos se han reportado previamente en frailejones con síntomas de diferentes páramos, información necesaria para establecer si los insectos pueden facilitar el ingreso y la colonización de fitopatógenos en la planta (Tack \& Dicke, 2013).

Dado que resulta indispensable comprender la dinámica del problema fitosanitario en E. pycnophylla, la presente investigación hace énfasis en la interacción entre insectos, hongos y frailejones, puesto que, se presume, que tanto la composición de insectos como de sus hongos asociados pueden variar, de acuerdo con el estado de salud de la población de frailejones (Ulyshen, 2016). En este sentido, los objetivos de la presente investigación fueron identificar cambios en las comunidades de insectos asociados a E. pycnophylla sin síntomas y con síntomas evidentes y evaluar si estos cambios en las comunidades de insectos se reflejan en los hongos asociados.

\section{MATERIALES Y MÉTODOS}

Área de estudio. El área de estudio corresponde a zonas del páramo de Paja Blanca con presencia de poblaciones sintomáticas, ubicadas en el municipio de Gualmatán (Nariño, Colombia) (Figura 1). Los límites altitudinales del páramo van desde los 3.200 a 3.650m. s.n.m., en los que domina vegetación achaparrada, asociaciones Pajonalmatorral, frailejonal y pajonal y las temperaturas medias anuales oscilan entre los 10,2 y 11, $6^{\circ} \mathrm{C}$ (Muñoz-Guerrero, 2017).

Colecta e identificación de insectos. El muestreo, se realizó en época seca del 2018, en cuatro zonas del páramo; en cada una, se seleccionaron seis frailejones sanos y seis con síntomas de clorosis, deformación y necrosis foliar. Los frailejones seleccionados presentaron alturas entre $70-90 \mathrm{~cm}$. En cada frailejón, se colectaron, de forma manual, insectos en estado adulto, tanto en la roseta como en la necromasa, los cuales, fueron dispuestos en viales plásticos estériles.

Los insectos, se identificaron hasta el nivel taxonómico más detallado posible, en los laboratorios de la Universidad de Nariño, mediante guías y claves taxonómicas (Triplehorn \& Johnson, 2004; Fernández \& Mason, 2006), comparación con material de referencia, que reposa en la Colección Entomológica de la Universidad de Nariño CEUN y la colaboración de especialistas.

Análisis ecológico. La diversidad alfa de los insectos colectados en frailejones sanos y con síntomas, se calculó con base en los números de Hill, donde la diversidad se expresa como ${ }^{\mathrm{q}} \mathrm{D}$, que se basa en el número efectivo de especies, donde q determina la influencia de la abundancia de las morfoespecies sobre los valores de diversidad. La diversidad de orden $\mathrm{q}=0$ representa la riqueza, la diversidad de orden $\mathrm{q}=1$, le da peso a las especies típicas y se calcula como el exponencial del índice de entropía de Shannon y la diversidad de orden $\mathrm{q}=2$, que le da mayor peso a las especies dominantes y se calcula con el inverso del índice de Simpson (Jost, 2006).

Para identificar el recambio de taxones entre los dos estados de frailejón evaluados, se aplicó el índice de similitud de Jaccard, mediante, el cual, es posible expresar el grado de semejanza de los individuos contenidos en dos muestras diferentes. Este coeficiente 

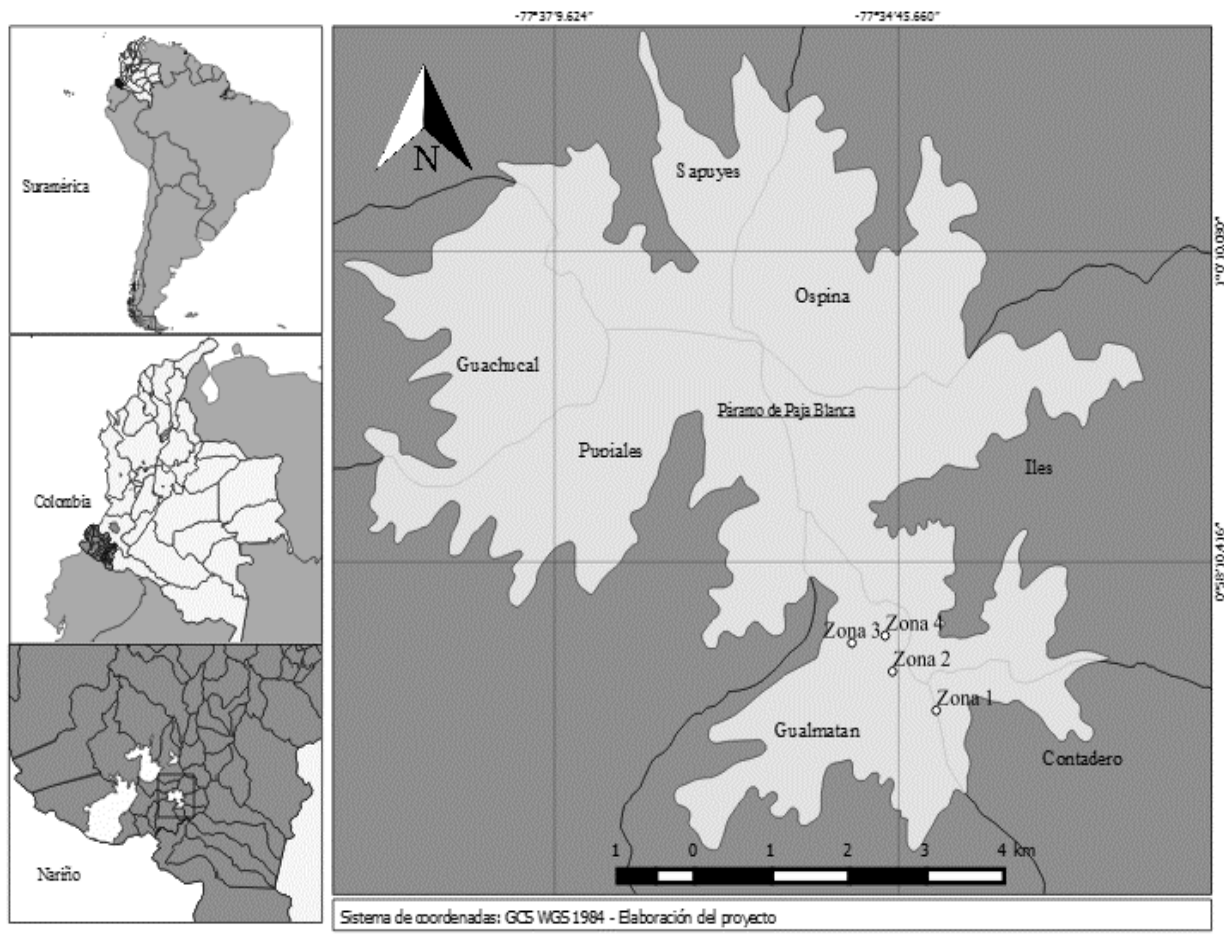

Figura 1. Localización del área de estudio y de las zonas de colecta, ubicadas en el páramo de Paja Blanca, Nariño (Colombia).

toma valores entre 0 , cuando las muestras no comparten taxones y 1, cuando tienen la misma composición de especies (Moreno et al. 2011). Estos análisis, se realizaron con el paquete "iNEXT" del programa estadístico R 3.3.0 (Chao et al. 2014).

Cultivo de hongos a partir de estructuras de insectos. De cada taxón de insecto, se extrajeron piezas bucales y el canal alimentario. Se maceraron con agua estéril y se sembró $0,1 \mathrm{~mL}$, en medio de cultivo PDA (agar dextrosa papa), con rifampicina. Los exoesqueletos de cada taxón de insecto, se lavaron con agua estéril en tubos Eppendorf y se llevaron a vortex, por un minuto. Del agua resultante del lavado, se sembró $0,1 \mathrm{~mL}$, en medio PDA. Todos los cultivos, se incubaron a $15^{\circ} \mathrm{C}$ y humedad relativa de $80 \%$ durante, aproximadamente, cinco días (Jaber et al. 2016).

Identificación morfológica y molecular de hongos. Las colonias emergentes se purificaron y tras su crecimiento, se identificaron hasta el nivel de género, mediante el uso de claves taxonómicas (Barnett \& Hunter, 1998; Cepero de García et al. 2012). A partir de cultivos monospóricos, se extrajo y se purificó $\mathrm{ADN}$, siguiendo el protocolo propuesto por Griffith \& Shaw (1998).

Para la amplificación de la región ITS del ADNr, se usaron los cebadores universales ITS1 e ITS4, descritos por White et al. (1990) y Álvarez-López et al. (2013). El volumen de la solución final para amplificación por PCR fue de $25 \mu \mathrm{L}(12,5 \mu \mathrm{L}$, de OneTaq Quick Load 2X Máster Mix/Standard Buffer - Promega; $1 \mu \mathrm{L}$ de cada cebador; $1 \mu \mathrm{L}$ de BSA y $2 \mu \mathrm{L}$ de la muestra de $\mathrm{ADN}(100 \mathrm{ng} / \mu \mathrm{L}))$. El producto, se visualizó por electroforesis en gel de agarosa $1,2 \%$ con $1 \mu \mathrm{L}$ de
Hydragreen ${ }^{\circledR}$. El gel, se corrió a 70V, durante 40 minutos. Los tamaños de las bandas, se compararon con un marcador de $100 \mathrm{pb}$ (Checa et al. 2015). Los productos de PCR, se secuenciaron por el método de Sanger, enviando las muestras al centro de investigación Corpogen (Bogotá, Colombia).

La búsqueda de identidad con otras secuencias reportadas en la base de datos de GenBank, se realizó mediante el programa BLAST (Basic Local Alignment Search Tool), del NCBI. Los hongos identificados fueron ingresados al cepario de la Universidad de Nariño.

Mediante revisión de literatura de los taxones de insectos identificados, las estructuras corporales y los hongos identificados, se relacionaron los resultados con el estado de E. pycnophylla del páramo de Paja Blanca.

\section{RESULTADOS Y DISCUSIÓN}

Análisis ecológico insectos. De los 276 individuos colectados, el $75 \%$ corresponde a los órdenes Diptera y Coleoptera y, la gran mayoría, estuvieron presentes, tanto en frailejones sanos como afectados (Tabla 1). Alzate (2010) afirma que la riqueza y abundancia de Coleoptera y Diptera, se debe a la alta disponibilidad de materia orgánica en descomposición aprovechable; asimismo, Amat-García \& Ríos-Vargas (1991) proponen que la dominancia de estos órdenes, se atribuye a las condiciones de humedad que presenta, tanto la roseta como la necromasa y que resultan adecuadas para los diferentes estados de desarrollo. 
Tabla 1. Identificación de insectos asociados a plantas de Espeletia pycnophylla sanas y afectadas, del páramo de Paja Blanca (Nariño, Colombia).

\begin{tabular}{|c|c|c|c|c|}
\hline \multicolumn{3}{|c|}{ Clasificación } & \multicolumn{2}{|c|}{ Número de individuos } \\
\hline Orden & Familia & Taxón & $\begin{array}{l}\text { Frailejones sin } \\
\text { síntomas }\end{array}$ & $\begin{array}{c}\text { Frailejones } \\
\text { afectados }\end{array}$ \\
\hline \multirow{10}{*}{ Coleoptera } & \multirow{2}{*}{ Carabidae } & Dyscolus sp. & 4 & 9 \\
\hline & & Lebia sp. & 0 & 3 \\
\hline & Scarabeidae & Uroxys sp. & 3 & 2 \\
\hline & Curculionidae & Entiminae & 5 & 3 \\
\hline & Anthribidae & Anthribidae & 0 & 5 \\
\hline & \multirow{2}{*}{ Chrysomelidae } & Diabrotica sp. 1 & 12 & 15 \\
\hline & & Diabrotica sp. 2 & 8 & 9 \\
\hline & Lampyridae & Lampyridae & 5 & 8 \\
\hline & \multirow{2}{*}{ Nitidulidae } & c. f Colopterus sp. & 5 & 8 \\
\hline & & c. f Carpopbilus sp. & 6 & 9 \\
\hline \multirow{10}{*}{ Diptera } & Tipulidae & Tipula sp. & 0 & 6 \\
\hline & Syrphidae & Copestylum sp. & 3 & 2 \\
\hline & Tephritidae & Neomyopites sp. & 9 & 15 \\
\hline & Muscidae & Muscidae & 5 & 8 \\
\hline & \multirow{2}{*}{ Tachinidae } & Tachinidae 1 & 3 & 6 \\
\hline & & Tachinidae 2 & 5 & 2 \\
\hline & Sarcophagidae & Sarcophagidae & 4 & 4 \\
\hline & Sciaridae & Bradysia sp. & 6 & 9 \\
\hline & Phoridae & Phoridae & 2 & 0 \\
\hline & Agromizydae & Ophiomyia sp. & 0 & 6 \\
\hline \multirow{4}{*}{$\begin{array}{l}\text { Hymenop- } \\
\text { tera }\end{array}$} & Ichneumonidae & Ichneumoninae & 2 & 0 \\
\hline & \multirow{2}{*}{ Apidae } & Bombus sp. 1 & 2 & 3 \\
\hline & & Bombus sp. 2 & 1 & 0 \\
\hline & Pteromalidae & Pteromalidae & 11 & 9 \\
\hline Hemiptera & Enicocephalidae & Enicocephalidae & 9 & 6 \\
\hline \multirow{2}{*}{ Blattodea } & \multirow{2}{*}{ Blattellidae } & Blattellidae 1 & 0 & 3 \\
\hline & & Blattellidae 2 & 6 & 9 \\
\hline \multicolumn{3}{|c|}{ Total } & 116 & 160 \\
\hline
\end{tabular}

Los valores de diversidad en términos de los números de Hill (q0, q1 y q2), para insectos colectados en frailejones sanos, fueron de $22,18,85$ y 16,6, respectivamente y de $25,21,218,8$, para frailejones afectados y de acuerdo con los intervalos de confianza, no se detectaron diferencias significativas (Figura 2). En consecuencia, la riqueza, la diversidad y la dominancia de taxones fue similar en los dos estados de frailejones muestreados, por lo que se infiere que los frailejones sintomáticos no se encuentran en un estado de daño en sus estructuras, que de paso a la colonización y la dominancia de insectos, propios de un estado de descomposición avanzado y que, por el contrario, aún mantienen condiciones adecuadas, que le permiten ser hábitat de diferentes taxones de insectos. La diversidad de la entomofauna puede ser un reflejo del estado de conservación en que se encuentran los frailejones, puesto que, en condiciones de baja intervención antrópica, donde la vegetación de páramo se ha desarrollado durante largos periodos de tiempo, la diversidad de la artropofauna encuentra una mayor homogeneidad ambiental y diversidad de recursos (Eraso-Puentes \& AmarilloSuárez, 2016).

El recambio de especies arrojó un valor de similitud de 0,7, de manera que no se identificó mayor variación en la composición de insectos, que se pueda atribuir al estado de afectación de los frailejones evaluados. Los resultados obtenidos contrastan con los de Camacho \& Marroquin (2016), en Espeletia lopezii, del Parque Nacional Natural el Cocuy, en donde se evaluó la composición de coleópteros, en tres estados fitosanitarios: muertos, enfermos y sanos, concluyendo que, en estado sano, existe una mayor diversidad 


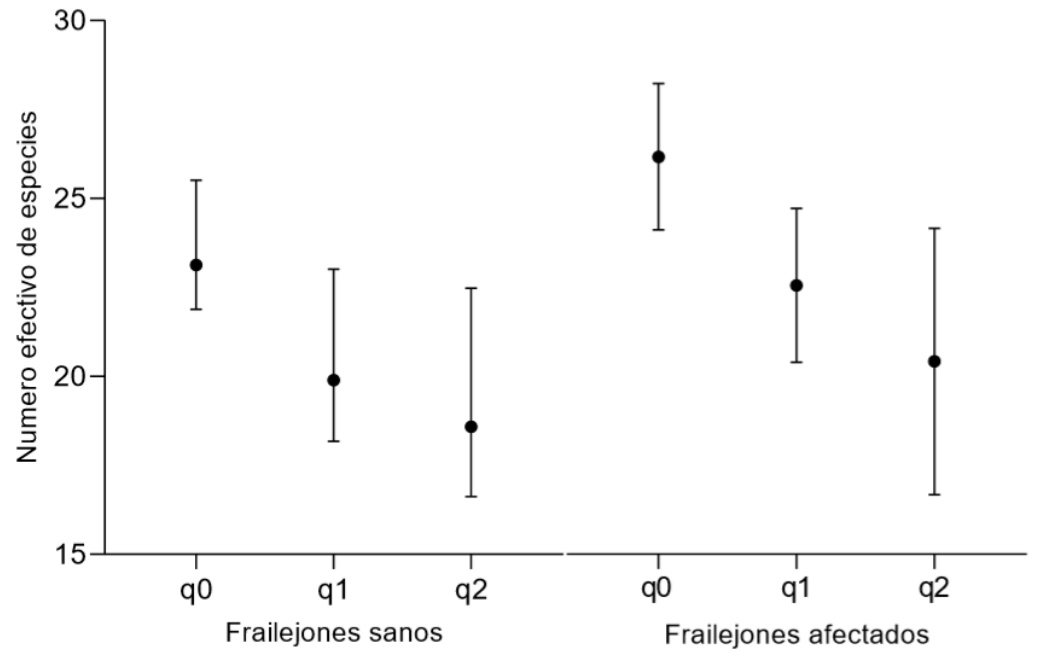

Figura 2. Perfiles de diversidad para frailejones sanos y afectados. Los puntos indican las medias y las líneas horizontales en cada intervalo representan los valores máximos y mínimos, con una significancia del 95\%.

de coleópteros, mientras que en el estado de descomposición más avanzado, hay dominancia de coleópteros Entiminae (Curculionidae) y Barinidae.
Hongos identificados. La identificación morfológica y molecular permitió reconocer 10 taxones hasta el nivel de especie y 11, a nivel de género (Tabla 2).

Tabla 2. Identidad, porcentaje de similitud, código de accesión (GenBank) y código de colección de los hongos aislados de diferentes estructuras de insectos asociados a frailejones sin síntomas y afectados, del páramo de Paja Blanca (Nariño, Colombia).

\begin{tabular}{|c|c|c|c|}
\hline Identidad GenBank & $(\%)$ identidad & Accesión & Código de colección \\
\hline Clonostachys sp. & 99 & KY413696 & I8LC02 \\
\hline Epicoccum nigrum & 98 & KR912314 & I2LC03 \\
\hline Gibberella thapsina & 99 & GU257900 & I6TG19 \\
\hline Epicoccum nigrum & 98 & KR912314 & $\mathrm{I} 2 \mathrm{AB} 03$ \\
\hline Rhizoctonia sp. & 99 & KJ395592 & $\mathrm{I} 3 \mathrm{AB} 14$ \\
\hline Cladosporium oryzae & 99 & KY400092 & I5LC20 \\
\hline Lecanicillium sp. & 99 & MH509414 & I6TG04 \\
\hline Phoma sp. & 99 & KT989564 & I5TG05 \\
\hline Metapochonia bulbillosa & 99 & MH483834 & I3AB21 \\
\hline Cadophora fastigiata & 99 & KP411560 & I5LC01 \\
\hline Sarocladium strictum & 99 & KY465763 & I8LC06 \\
\hline Talaromyces variabilis & 96 & KT310940 & I4LC07 \\
\hline Fusarium oxysporum & 99 & MF435919 & I1LC08 \\
\hline Microdochium sp. & 98 & KU901555 & I4TG09 \\
\hline Botrytis sp. & 99 & KF015582 & I4TG13 \\
\hline Acremonium murorum & 99 & HQ637278 & I3LC18 \\
\hline Microdochium sp. & 98 & MK163888 & I1LC09 \\
\hline Epicoccum nigrum & 98 & KR912314 & $\mathrm{I} 3 \mathrm{AB} 03$ \\
\hline Trichoderma sp. & 99 & MH284582 & I2TG10 \\
\hline
\end{tabular}


Los géneros Paecilomyces sp., Penicillium sp. y Trichoderma sp., se reportan en E. grandiflora y tienen capacidad biocontroladora sobre fitopatógenos, como Fusarium oxysporum (Miles et al. 2012). Epicoccum, endófito de Espeletia spp. (Cabezas et al. 2012; Varela, 2014; FloresBazauri et al. 2015), evidencia potencial biocontralador sobre $F$. oxysporum y Rhizoctonia solani (Ogórek \& Plaskowsk, 2011). Talaromyces variabilis, se emplea en la producción de antibióticos (Dutta et al. 2014), Clonostachys sp. tiene capacidad de inhibir in vitro el crecimiento de F. oxysporum y Phoma sp. y Mucor sp., se han aislado de Espeletia grandiflora y pueden ser empleados como potenciales controladores de hongos fitopatógenos (Anacona et al. 2005).

Fusarium sp. contiene especies endófitas y fitopatógenas. Algunas especies endófitas tienen potencial para ser utilizados como controladores biológicos de insectos u otros hongos, mientras que las especies fitopatógenas generan pérdidas económicas significativas, debido al devastador impacto que pueden tener en las cosechas (Villa-Martínez et al. 2015); por ejemplo, Fusarium oxysporum, causante de marchitez vascular en una amplia gama de cultivos (VásquezRamírez \& Castaño-Zapata, 2017).

Botrytis sp. causa estrangulamiento del tallo, manchas y pudrición de raíces, se asocia con la pudrición foliar de Espeletia grandiflora (García-Castañeda et al. 2015) y al igual que Cladosporium sp., es un posible causante de la pérdida de pubescencia foliar en Espeletia paipana (Buitrago et al. 2015). Rhizoctonia sp. ataca a las plantas de cultivo, a nivel de las raíces, generando manchas necróticas, que se desarrollan hasta los tallos y los pecíolos de las hojas, causando la caída y muerte de la planta (Lahlali \& Hijri, 2010).

Relación entre hongos aislados y estructuras corporales de insectos. Se logró aislar un total de 17 taxones de hongos entre géneros y especies, a partir del aparato bucal, el canal alimentario y exoesqueleto de insectos, presentes en frailejones afectados (Tabla 3) y 11 taxones de hongos entre géneros y especies, a partir de las estructuras mencionadas de insectos, presentes en frailejones sin síntomas (Tabla 4).

Tabla 3. Hongos aislados de estructuras de insectos presentes en plantas de E. pycnophylla afectadas, del páramo de Paja Blanca (Nariño). A.B = aparato bucal; C.A canal alimentario y L.C=lavado del cuerpo.

\begin{tabular}{|c|c|c|c|c|c|c|c|c|c|c|c|c|c|c|c|c|c|c|c|c|c|}
\hline & \multicolumn{3}{|c|}{ Neomyopites } & \multicolumn{3}{|c|}{ Dyscolus } & \multicolumn{3}{|c|}{$\begin{array}{c}\text { Diabrotica } \\
\text { sp. } 1\end{array}$} & \multicolumn{3}{|c|}{$\begin{array}{c}\text { Diabrotica } \\
\text { sp. } 2\end{array}$} & \multicolumn{3}{|c|}{$\begin{array}{c}\text { c.f } \\
\text { Carpopbilus }\end{array}$} & \multicolumn{3}{|c|}{ Bradysia } & \multicolumn{3}{|c|}{ Tachinidae 1} \\
\hline & L.C & A.B & C.A & L.C & A.B & C.A & L.C & A.B & C.A & L.C & A.B & C.A & L.C & A.B & C.A & L.C & A.B & C.A & L.C & A.B & C.A \\
\hline Clonostachys sp. & & & & & & & & & & & & & & & & $\mathrm{x}$ & & & & & \\
\hline E. nigrum & $\mathrm{x}$ & & & & & & & $\mathrm{x}$ & & & & & & & & & & & & & \\
\hline Lecanicillium sp. & & & & $\mathrm{x}$ & & & & & & & & & & & & & $\mathrm{x}$ & & & & \\
\hline Phoma sp. & & & $\mathrm{x}$ & & & & & & & & & & & & $\mathrm{x}$ & & & & & & \\
\hline S. strictum & & & & & & & & & & & & & & & & $\mathrm{x}$ & & & & & \\
\hline T. variabilis & & $\mathrm{x}$ & & & & & $\mathrm{x}$ & & & $\mathrm{x}$ & & & & & & & & & & & \\
\hline F. oxysporum & & $\mathrm{x}$ & & & & & & $\mathrm{x}$ & & & & & & & $\mathrm{x}$ & $\mathrm{x}$ & & & & & \\
\hline Microdocbium sp. & $\mathrm{x}$ & & & & & & & & & & & $\mathrm{x}$ & & & & & & & & & \\
\hline Trichoderma sp. & & & & & $\mathrm{x}$ & & & & & & & $\mathrm{x}$ & & & & & & & & & \\
\hline Penicillium sp. 1 & $\mathrm{x}$ & & & & $\mathrm{x}$ & & $\mathrm{x}$ & & & & & & & & & & & & & $\mathrm{x}$ & \\
\hline Penicillium sp. 2 & & & & & & & & & & & & & & & & $\mathrm{x}$ & & & & $\mathrm{x}$ & \\
\hline Botrytis sp. & & & & & & & & & & & & & $\mathrm{x}$ & & & $\mathrm{x}$ & & & & & \\
\hline Rbizoctonia sp. & & & & & & & & $\mathrm{x}$ & & & & & & & & & & & & & \\
\hline Paecilomyces sp. & & $\mathrm{x}$ & & & & & & & & & & & & & & & & & & & \\
\hline Fusarium sp. & $\mathrm{x}$ & $\mathrm{x}$ & & & & & & & & & & & & & & & & & & & \\
\hline A. murorum & & & & & & & $\mathrm{x}$ & & & & & & & & & & & & & & \\
\hline Mucor sp. & & & & & & & & & & $\mathrm{x}$ & & & & & & & & & & & \\
\hline
\end{tabular}


Tabla 4. Hongos aislados de estructuras de insectos presentes en plantas de E. pycnophylla sin afectación, del páramo de Paja Blanca (Nariño). A.B $=$ aparato bucal; $C . A=$ canal alimentario y $\mathrm{L} . \mathrm{C}=$ lavado del cuerpo.

\begin{tabular}{|c|c|c|c|c|c|c|c|c|c|c|c|c|c|c|c|c|c|c|c|c|c|}
\hline & \multicolumn{3}{|c|}{ Neomyopites } & \multicolumn{3}{|c|}{ Dyscolus } & \multicolumn{3}{|c|}{ Diabrotica sp. 1} & \multicolumn{3}{|c|}{$\begin{array}{l}\text { Diabrotica } \\
\text { sp. } 2\end{array}$} & \multicolumn{3}{|c|}{ c.f Carpophilus } & \multicolumn{3}{|c|}{ Colopterus } & \multicolumn{3}{|c|}{ Bombus sp. 1} \\
\hline & L.C & A.B & C.A & L.C & A.B & C.A & L.C & A.B & C.A & L.C & A.B & C.A & L.C & A.B & C.A & L.C & A.B & C.A & L.C & A.B & C.A \\
\hline Lecanicillium sp. & & & & & & & $\mathrm{x}$ & & & & & & & & $\mathrm{x}$ & $\mathrm{x}$ & & & & & \\
\hline F. oxysporum & $\mathrm{x}$ & & & & & & & & & & & & & & & & & & & & \\
\hline Penicillium sp.1 & & & $\mathrm{x}$ & & & $\mathrm{x}$ & $\mathrm{x}$ & & & & & & & & & & & $\mathrm{x}$ & & $\mathrm{x}$ & \\
\hline Botrytis sp. & $\mathrm{x}$ & & & & & & & & & & & $\mathrm{x}$ & & & & & & & & & \\
\hline Paecilomyces sp. & & & $\mathrm{x}$ & $\mathrm{x}$ & & & & & & & & & & & & & & & & & \\
\hline G. thapsina & & & & & & & & & & $\mathrm{x}$ & & & & & & & & $\mathrm{x}$ & & & \\
\hline
\end{tabular}

El aparato bucal chupador del género Neomyopites le permite absorber líquidos de las frutas, los carbohidratos y los nutrientes, que son liberados por las hojas de las plantas. La alimentación, a través de una probóscide, implica el contacto de esta estructura con el sustrato que, en el caso de encontrarse contaminado, puede facilitar la dispersión de hongos (Coronado-Gonzalez et al. 2009).

Especies del género Dyscolus sp., se reportan en Espeletia argentea, del Parque Nacional Natural Páramo de Chingaza, ingresando a la planta por cavidades que construyen en la base de la roseta, produciendo daño a hojas maduras. Posiblemente, la presencia de fitopatógenos en su aparato bucal, se deba al consumo de otros invertebrados, como Collembola, larvas de Lepidoptera y Psocoptera, que se pudieron alimentar de tejidos de plantas afectadas y cuyas esporas presentes en el cuerpo pudieron entrar en contacto con las mandíbulas del depredador (Matta et al. 2017).

Los géneros de insectos con aislamientos de fitopatógenos, a partir de lavados de cuerpo, fueron Neomyopites, Dyscolus y Bradysia. Neomyopites sp. no presenta reportes como trasmisor mecánico; sin embargo, cabe mencionar el caso de Anastrepha fraterculus, que pertenece a la misma tribu Myopitinii, cuyas hembras ovopositan en frutos de uva, facilitando el ingreso de fitopatógenos, que causan la caída del fruto, como Cladosporium spp., Botrytis cinerea, Fusarium spp. y Rhizopus spp., que se aislaron a partir de patas, alas, cabeza y ovopositor (Machota et al. 2013). Posiblemente, las setas en el cuerpo de Neomyopites sp., igualmente, faciliten la adhesión de esporas de hongos y su posterior dispersión.

La capacidad de transportar patógenos de Bradysia sp., se ha documentado en Eucalyptus spp., donde las plántulas son afectadas, tanto por herbivoría de larvas como por la dispersión de hongos patógenos. El tipo de microorganismo que es transmitido, depende del estado de desarrollo de Bradysia sp., de manera que los adultos pueden transportar, en su cuerpo, conidias de hongos patógenos, que producen estadios de dispersión aérea, como $B$. cinerea, $F$. oxysporum que, luego, se pueden transmitir a plantas sanas (Gillespie \& Menzies, 1993; Santos et al. 2012).

Shamshad et al. (2009) explican que Bradysia ocellaris tiene mayor capacidad de carga de esporas que Lycoriella ingenua (Sciaridae), ya que, la primera, presenta una hilera de cerdas a manera de peine en las tibias delanteras, que facilitan la adhesión de esporas.

Como se ha mencionado, Dyscolus sp. se puede encontrar en Espeletia sp. depredando otros artrópodos; la explicación de la presencia de fitopatógenos se puede argumentar, teniendo en cuenta que la capacidad de carga de inóculo está relacionada, además, con el tamaño del individuo, el área de superficie y la anatomía del insecto. Teniendo en cuenta que entre los insectos colectados Dyscolus sp. es el de mayor tamaño, se puede esperar que tenga esporas asociadas a su cuerpo (Schweigkofler et al. 2005).

El aislamiento de hongos a partir del canal alimentario es una aproximación a la capacidad de los hongos de mantenerse viables en el interior de los insectos y a que, posiblemente, se puedan mantener infectivas en sus deposiciones que, de llevarse a cabo sobre plantas sanas, contribuiría a la dispersión de hongos fitopatógenos, tal como ocurre en larvas del género Bradysia, en las que se ha documentado la capacidad de consumo de tejidos de raíz afectados con fitopatógenos, como el Oomycete Pythium sp., cuyas oosporas pueden sobrevivir en el canal alimentario y mantenerse viables después del proceso de excreción (Hyder et al. 2009). 
El estudio de la diversidad de insectos en ecosistemas de páramo aporta al conocimiento de este grupo en regiones poco exploradas, permitiendo reconocer que hay una gran diversidad taxonómica y que existe la necesidad de profundizar en los inventarios, abordando diferentes variables, que permitan tener un mayor entendimiento sobre posibles patrones en la distribución de insectos que aporten información, que pueda ser utilizada en la formulación y en la ejecución de estrategias encaminadas a la conservación de ecosistemas de gran importancia, como son los páramos.

Se recomienda plantear, a futuro, investigaciones que profundicen en el papel de los insectos en la dispersión de fitopatógenos, partiendo de las reglas para determinar si un insecto es vector de un patógeno vegetal, propuestas por Leach (1940), que plantean, en primer lugar, una asociación estrecha entre el insecto con la planta enferma; segundo, registrar si el insecto debe realiza visitas periódicas a plantas sanas; tercero, el insecto debe estar asociado con el patógeno que causa la enfermedad en cuestión y, cuarto, confirmar que la visita de plantas sanas por insectos infestados de patógenos resulta en el desarrollo de la enfermedad.

Agradecimientos. A la Doctora Melba Santamaría Ruano y al Biólogo Mauricio Rodríguez, por la colaboración durante el proceso de identificación de insectos y a los Doctores Tito Bacca y Amanda Varela, por sus aportes. A la Corporación Autónoma Regional de Nariño Corponariño, al Centro de estudios ambientales de la Facultad de Ciencias de la Universidad de Nariño CEA y la Vicerrectoría de investigaciones, postgrados y relaciones internacionales VIPRI, de la Universidad de Nariño, por la financiación de esta investigación. Conflicto de intereses. Los autores declaran no tener ningún conflicto de interés.

\section{REFERENCIAS}

1. ÁlVAREZ-LÓPEZ, C.; OSORIO-VEGA, O.; MARÍNMONTOYA, M. 2013. Identificación molecular de microorganismos asociados a la rizosfera de plantas de vainilla en Colombia. Acta Biol. Col. 18(2):293-305.

2. ALZATE-QUINTERO, N.F. 2010. Insectos asociados a la necromasa de frailejón (Espeletia hartwegiana Cuatrec), en un páramo de Villamaría, Caldas. Agronomía (Manizales). 18(1):59-68.

3. AMAT-GARCÍA, G.; RÍOS-VARGAS, O. 1991. Caracterización de Microhábitats de la Artropofauna en páramos del Parque Nacional Natural Chingaza Cundinamarca, Colombia. Caldasia. 16(79):539-550.

4. ANACONA, A.; SABOGAL, S.; GARCÉS, E. 2005. Distribución de las especies de hongos asociadas al abrigo de hojas muertas de Espeletia grandiffora, en el páramo El Granizo. En: Bonilla M, Editor. Estrategias adaptativas de plantas del páramo y del bosque altoandino en la cordillera oriental de Colombia. Bogotá: Unibiblos. p.107-122.
5. BARNETT, H.L.; HUNTER, B.B. 1998. Illustrated genera of imperfect fungi. (4th ed). Amer Phytopathological Society APS Press. 240p.

6. BUITRAGO, S.; VANEGAS, L.; RAMOS, C. 2015. Pérdida de pubescencia foliar y sus efectos fisiológicos en Espeletia paipana (Asterales, Asteraceae), en el departamento de Boyacá-Colombia. Rev. Biol. Trop. 63(3):845-858. https://doi.org/10.13140/RG.2.1.4748.2723

7. CABEZAS, L.; CALDERON, C.; MEDINA, L.M.; BAHAMON, I.; CARDENAS, M.; BERNAL, A.J.; GONZALEZ, A.; RESTREPO, S. 2012. Characterization of cellulases of fungal endophytes isolated from Espeletia spp. Res.J. Microbiol. 50(6):1009-1013. https://doi.org/10.1007/s12275-012-2130-5

8. CAMACHO, M.; MARROQUIN, J. 2016. Análisis de insectos Coleoptera asociados a la afectación del frailejón Espeletia lopezzii en el páramo de la vertiente occidental del Parque Nacional Natural el Cocuy. In Situ. 3:5-15.

9. CEPERO DE GARCÍA, M.C.; RESTREPO, S.; FRANCO, A.E.; CÁRDENAS, M.E.; VARGAS, N. 2012. Hongos anamórficos, conidiales, mitospóricos o 'deuteromycetes. En: Cepero De García, M.C.; Restrepo Restrepo, S.; FrancoMolano, A.E.; Cárdenas Toquica, M.; Vargas Estupiñan, N. (eds) Biología De Hongos. 1st ed., Universidad De Los Andes, Colombia, Bogotá, D.C., Colombia. p.119-162.

10. CHAO, A.; GOTELLI, N.J.; HSIEH, T.C.; SANDER, E.L.; MA, K.H.; COLWELL, R.K.; ELLISON, A.M. 2014. Rarefaction and extrapolation with Hill numbers: a framework for sampling and estimation in species diversity studies. Ecological Monographs. 84(1):45-67. https://doi.org/10.1890/13-0133.1

11. CHECA, O.E.; DESCANCE, J.A.; TORO, M.X.; ÁLVAREZ, S.L.; SALAZAR, C. 2015. Caracterización molecular de Trichoderma spp. en arveja Pisum sativum L. Revista de Ciencias Agrícolas. 32(2):03-12.

https://dx.doi.org/10.22267/rcia.153202.8

12. CORONADO-GONZALEZ, P.A.; VIJAY-SEGARAN, S.; ROBINSON, A.S. 2009. Functional Morphology of the mouthparts of the Adult Mediterranean Fruit Fly, Ceratitis capitata. J. Insect Sci. 8(73):1-11. https://doi.org/10.1673/031.008.7301

13. DUTTAA, D.; PUZARI, K.C.; GOGOI, R.; DUTTA, P. 2014. Endophytes: exploitation as a tool in plant protection. Braz. Arch. Biol. Technol. 57:621-629.

http://dx.doi.org/10.1590/S1516-8913201402043

14. ERASO-PUENTES, L.P.; AMARILLO-SUÁREZ, Á.R. 2016. Arthropods in necromass of two rosette plants species in 
different successional stages of Andean Páramo. Rev. Col. Entomol. 42(1):81-90.

https:/ / doi.org/10.25100/ socolen.v42i1.6674

15. FERNÁNDEZ, F.; MASON, W. 2006. Introducción a los de Hymenoptera de la región neotropical. En: Fernández, F; Sharkey, M.J. (eds.) Introducción a los Hymenoptera de la Región Neotropical. Universidad Nacional de Colombia. Sociedad Colombiana de Entomología SOCOLEN. Bogotá, Colombia. 893p.

16. FLORES-BAZAURI，W.; CHICO-RUÍZ, J.; CERNAREBAZA, L.C. 2015. Actividad antagónica in vitro de Clonostachys rosea sobre Fusarium oxysporum, Alternaria solani y Botrytis cinerea. Revista Rebiol. 35(1):34-42.

17. GARCÍA-CASTAÑEDA, A.R.; DÍAZ-ALVARADO, Á.I.; CASTAÑEDA-GARZÓN, S.L.; CELESTINO, J.B. 2015. Diagnóstico preliminar de microorganismos fitopatógenos asociados a plántulas de Espeletia grandiflora Humb. \& Bonpl. Propagadas para su conservación Ex situ. Fitopat. Col. 39(1):14

18. GILLESPIE, D.R.; MENZIES, J.G. 1993. Fungus gnats vector Fusarium oxysporum f. sp. radicislycopersici. Ann. Applied Biology. 123(427):539-544. https://doi.org/10.1111/j.1744-7348.1993.tb04926.x

19. GRIfFITH, G.W.; SHAW, D.S. 1998. Polymorphisms in Phytophthora infestans: four mitochondrial haplotypes are detected after PCR amplification of DNA from pure cultures or from host lesions. Appl. Environ. Microbiol. 64(10):4007-4014. https://doi.org/10.1128/AEM.64.10.4007-4014.1998

20. HYDER, N.; COFFEY, M.D.; STANGHELLINI, M.E. 2009. Viability of Oomycete Propagules Following Ingestion and Excretion by Fungus Gnats, Shore Flies, and Snails. Plant Disease. 93(7):720-726. https://doi.org/10.1094/pdis-93-7-0720

21. JABER, S.; MERCIER, A.; KNIO, K.; BRUN, S.; KAMBRIS, Z. 2016. Isolation of fungi from dead arthropods and identification of a new mosquito natural pathogen. Parasit Vectors. 9(1):491. https://doi.org/10.1186/s13071-016-1763-3

22. JOST, L. 2006. Entropy and diversity. Oikos. 113(2):363-375. https://doi.org/10.1111/j.2006.0030-1299.14714.x

23. LAHLALI, R.; HIJRI, M. 2010. Screening, identification and evaluation of potential biocontrol fungal endophytes against Rhizoctonia solani AG3 on potato plants. FEMS Microbiology Letters. 311(2):152-159.

https://doi.org/10.1111/j.1574-6968.2010.02084.x
24. LEACH, J.G. 1940. Insect Transmission of Plant Diseases. Journal of the New York Entomological Society. 48:403404.

25. MACHOTA, J.R.; BORTOLI, L.C.; BOTTON, M.; GRÜTZMACHER, A.D. 2013. Fungi that cause rot in bunches of grape identified in adult fruit flies (Anastrepha fraterculus) (Diptera: Tephritidae). Chil. J. Agric. Res. 73(2):34-35.

https://doi.org/10.4067/s0718-58392013000200018

26. MATTA, D.H.; CIVIDANES, F.J.; SILVA, R.J.; BATISTA, M.N.; OTUKA, A.K.; CORREIA, E.T.; MATOS, S.T. 2017. Feeding habits of Carabidae (Coleoptera) associated with herbaceous plants and the phenology of coloured cotton. Acta Scientiarum. Agronomy. 39(2):135-142. https://doi.org/10.4025/actasciagron.v39i2.32593

27. MILES, L.A.; LOPERA, C.A.; GONZÁLEZ, S.; CEPERO DE GARCÍA, M.C.; FRANCO, A.E.; RESTREPO, S. 2012. Exploring the biocontrol potential of fungal endophytes from an Andean Colombian Paramo ecosystem. BioControl. 57(5):697-710.

https://doi.org/10.1007/s10526-012-9442-6

28. MORENO, C.E.; BARRAGÁN, F.; PINEDA, E.; PAVÓN, N. 2011. Reanalyzing alpha diversity: alternatives to understand and compare information about ecological communities. Revista Mexicana de Biodiversidad. 82(1):1249-1261.

29. MUÑOZ-GUERRERO, D.A. 2017. Transformaciones y prospectiva del paisaje en el páramo de Paja Blanca, Nariño, Colombia. Persp. Geog. 22(2):47-66. https://dx.doi.org/10.19053/01233769.7598

30. NÁRDIZ, P.M.; DE CAL, A. 2006. Biofungicidas y control biológico de hongos fitopatógenos: aplicación en la filosfera. Phytoma España. 182:59-63.

31. OGÓREK, R.; PLASKOWSK, E. 2011. Epicoccum nigrum for biocontrol agents in vitro of plant fungal pathogens. Commun. Agric. Appl. Biol. Sci. 76(4):691-697.

32. SALINAS, C.; FUENTES, L.S.; HERNÁNDEZ, L. 2013. Caracterización de los lepidópteros fitófagos asociados a la herbivoría de frailejones en la microcuenca de la quebrada Calostros del Parque Nacional Natural Chingaza. Revista Mutis. 3(1):1-22. https://doi.org/10.21789/22561498.838

33. SANTOS, A.; ZANETTI, R.; ALMADO, R.P.; SERRÃO, J.E.; ZANUNCIO, J.C. 2012. First report and population changes of Bradysia difformis (Diptera: Sciaridae) on Eucalyptus nurseries in Brazil. Fla. Entomol. 95(3):569-572. https://doi.org/10.1653/024.095.0305 
34. SCHWEIGKOFLER, W.; OTROSINA, W.J.; SMITH, S.L.; CLUCK, D.R.; MAEDA, K.; PEAY, K.G.; GARBELOT'TO, M. 2005. Detection and quantification of Leptographium wageneri the cause of black-stain root disease, from bark beetles (Coleoptera: Scolytidae) in Northern California using regular and real-time PCR. Can. J. For. Re. 35(8):17981808.

https://doi.org/10.1139/x05-077

35. SHAMSHAD, A.; CLIFT, A.D.; MANSFIELD, S. 2009.The effect of tibia morphology on vector competency of mushroom sciarid flies. J. Appl. Entomol. 133(6):484-490. https://doi.org/10.1111/j.1439-0418.2008.01362.x

36. TACK, A.M.; DICKE, M. 2013. Plant pathogens structure arthropod communities across multiple spatial and temporal scales Funct. Ecol. 2013:633-645. https://doi.org/10.1111/1365-2435.12087

37. TRIPLEHORN, C.H.; JOHNSON, N. 2004. Borror and DeLong's Introduction to the Study of Insects. 7th ed. Brooks/Cole, Ed. CA, United States. Cengage Learning, Inc. $888 \mathrm{p}$.

38. ULYSHEN, M.D. 2016. Wood decomposition as influenced by invertebrates. Biolog. Reviews. 91(1):70-85.

https://doi.org/10.1111/brv.12158
39. VARELA, A. 2014. Limitantes en la restauración ecológica: Estudio de caso de las afectaciones por patógenos en el parque Nacional Natural Chinagaza. En: Cubides, P.J.; Ramírez, W.; Cabrera, M.; Aguilar-Garavito, M.; Fajardo-Gutiérrez, F; Vargas, W. eds. 1st ed. Restauración ecológica de los páramos de Colombia, Transformación y herramientas para su conservación. 296p.

40. VÁSQUEZ-RAMÍREZ, L.; CASTAÑO-ZAPATA, J. 2017. Manejo integrado de la marchitez vascular del tomate [Fusarium oxysporum f. sp. lycopersici (SACC.) W.C. Snyder \& H.N. Hansen]: una revisión. Rev. U.D.C.A Act. \& Div. Cient. 20(2):363-374.

https://doi.org/10.31910/rudca.v20.n2.2017.394

41. VILLA-MARTÍNEZ, A.; PÉREZ-LEAL, R.; MORALESMORALES, H.A.; BASURTO-SOTELO, M.; SOTOPARRA, J.M.; MARTÍNEZ-ESCUDERO, E. 2015. Situación actual en el control de Fusarium spp. y evaluación de la actividad antifúngica de extractos vegetales. Acta Agronómica. 64(2):194-205. https://doi.org/10.15446/acag.v64n2.43358

42. WHITE, T.; BRUNS, T.; LEE, S.; TAYLOR J. 1990. Amplification and direct sequencing of fungal ribosomal RNA genes for phylogenetics. In: Innis, M.A.; Gelfand, D.H.; Sninsky, J.J.; White, T.J. (eds.). PCR Protocols. A guide to methods and applications. Academic Press. London, England. p.315-322. https://doi.org/10.1016/B978-0-12-372180-8.50042-1 